\title{
Un diplôme pour l'aumônerie des services publics
}

\section{Francis Messner et Pierre-Henri Prélot}

\section{OpenEdition}

Journals

Édition électronique

URL : https://journals.openedition.org/rdr/632

DOI $: 10.4000 /$ rdr.632

ISSN : 2534-7462

\section{Éditeur}

Presses universitaires de Strasbourg

\section{Édition imprimée}

Date de publication : 31 octobre 2017

Pagination : 181-193

ISBN : $978-2-86820-974-0$

ISSN : 2493-8637

\section{Référence électronique}

Francis Messner et Pierre-Henri Prélot, "Un diplôme pour l'aumônerie des services publics », Revue du droit des religions [En ligne], 4 | 2017, mis en ligne le 15 janvier 2020, consulté le 23 mai 2022. URL http://journals.openedition.org/rdr/632; DOI : https://doi.org/10.4000/rdr.632

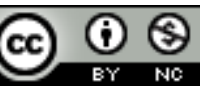

La revue du droit des religions est mise à disposition selon les termes de la Creative Commons Attribution - Pas d'Utilisation Commerciale 4.0 International - CC BY-NC 4.0. 


\section{UN DIPLÔME POUR L'AUMÔNERIE DES SERVICES PUBLICS}

\section{Francis MESSNER et Pierre-Henri PRÉLOT}

Université de Strasbourg / CNRS (DRES) ; Université de Cergy-Pontoise

es échéances électorales nationales sont généralement l'occasion pour
le pouvoir sortant de publier à la chaîne, dans les derniers jours de son existence, de nombreux textes réglementaires jusqu'ici restés en instance. C'est le cas pour le décret n 2017-756 du 3 mai 2017 relatif aux aumôniers militaires, hospitaliers et pénitentiaires et à leur formation civile et civique, et de l'arrêté interministériel du 5 mai 2017 relatif aux diplômes de formation civile et civique suivie par les aumôniers militaires d'active et les aumôniers hospitaliers et pénitentiaires et fixant les modalités d'établissement de la liste de ces formations. Il s'agit de deux textes auxquels le ministre de l'Intérieur Bernard Cazeneuve, devenu en décembre 2016 Premier ministre, était très attaché, parce que l'urgence de la lutte contre le radicalisme et le terrorisme islamistes en commandait l'adoption, en particulier s'agissant des aumôniers intervenant en milieu carcéral.

La présentation de ces dispositions impose d'analyser en premier lieu le nouveau dispositif de formation à la laïcité (1). L'objet de la seconde partie de cette contribution est d'expliquer la manière dont ce dispositif de formation s'inscrit dans le cadre juridique complexe qui organise l'aumônerie, ce qui conduira à souligner que la possession du diplôme n'est pas une condition de l'accès aux fonctions d'aumônier, mais une condition de la rémunération (2). Enfin, on s'attachera dans une troisième partie à présenter les spécificités 
propres à chaque catégorie d'aumônerie ainsi que les dispositions transitoires prévues par le décret (3).

\section{LE DISPOSITIF DE FORMATION CIVILE ET CIVIQUE}

On se propose ici de présenter le diplôme de formation civile et civique ainsi que le contenu de la formation (1.2), après en avoir rappelé les origines (1.1).

\subsection{AUX ORIGINES DE LA FORMATION CIVILE ET CIVIQUE}

La création d'une formation civile et civique obligatoire pour l'ensemble des aumôniers de l'armée et des établissements pénitentiaires et hospitaliers rémunérés trouve son origine dans une réflexion plus large menée par les pouvoirs publics sur la formation des cadres religieux musulmans. En effet, une partie importante des cadres permanents de cette religion sont formés à l'étranger et connaissent mal le contexte français. Si aujourd'hui l'attention se porte pour des raisons d'évidence sur l'islam, certains prêtres catholiques, rabbins ultra-orthodoxes ou encore pasteurs évangéliques pourraient faire l'objet d'un constat similaire.

Les autorités publiques ont dès les années 1990 engagé une réflexion sur la formation des cadres religieux dont font partie les aumôniers, ce qui a favorisé la création de l'Institut d'études de l'islam et des sociétés du monde musulman (IISMM/École des hautes études en sciences sociales) par le ministère de l'Éducation nationale, de la Recherche et de la Technologie en 1999. Cette question a été à nouveau évoquée par le ministre de l'Intérieur Nicolas Sarkozy le 29 mars 2003. S'exprimant devant le Conseil des imams de France, il a alors soutenu que « les imams doivent être formés en France, connaître notre pays, ses traditions, ses lois [...] », tout en soulignant qu'il appartient aux communautés musulmanes de préciser leurs besoins de formation théologique. Finalement, le ministre de l'Éducation nationale a demandé un rapport à Daniel Rivet, directeur de l'IISMM, aux fins de préciser quel pourrait être l'apport de l'Université publique à la formation des imams. Une commission interministérielle a par ailleurs été créée (Premier ministre, ministre de l'Intérieur et ministre des Affaires étrangères). Suite à un débat tendu sur le contenu de cette formation, les décideurs ont in fine retenu la nécessité de créer une formation à l'intégration des ministres 
du culte musulman par le biais d'un diplôme universitaire (DU) dont le contenu devait se focaliser sur l'apprentissage de la langue française et la connaissance des institutions et des lois de la République. Cette solution permettait d'écarter l'hypothèse, embarrassante en régime de laïcité, de la création d'une filière publique de formation théologique des cadres religieux musulmans.

Ce DU devait, à la demande du recteur de l'académie de Paris, être pris en charge par l'Université Paris IV avec le soutien de l'Université Paris II pour tout ce qui concerne les enseignements de droit. La demande de création de ce DU a cependant été repoussée par le conseil des études et de la vie universitaires (CEVU) de Paris II. La formation préconisée par la commiswsion interministérielle a toutefois été créée et prise en charge en 2008 par la Faculté de sciences sociales et économiques (FASSE) de l'Institut catholique de Paris, établissement privé d'enseignement supérieur, sous la forme d'un diplôme intitulé "Interculturalité, laïcité, religion».

Le premier DU créé dans une université publique remonte à 2010. Il s'agit du diplôme d'université « Droit, société et pluralité des religions » de la Faculté de droit de l'Université de Strasbourg. Quant au troisième DU, créé en 2012 sous l'impulsion du préfet de la Région Rhône-Alpes, il constitue un modèle intéressant, dans la mesure où il associe trois partenaires, l'Université catholique de Lyon, la Faculté de droit de l'Université Lyon 3 et l'Institut français de civilisation musulmane (IFCM) de la Grande Mosquée de Lyon. Ce DU «Religion, liberté religieuse et laïcité » est délivré à des étudiants fonctionnaires ou agents publics, l'IFCM délivrant quant à lui un certificat "Connaissance de la laïcité " à des imams ou à des responsables d'associations cultuelles ou culturelles musulmanes. À partir de la rentrée 2017, le DU sera délivré à tous les étudiants. La même année, la faculté de droit de l'Université Montpellier 1 a instauré un DU « Religion et société démocratique ». Quant à l'IEP d'Aix-en-Provence, il a ouvert son DU «Pluralité religieuse, droit, laïcités et sociétés » en janvier 2014. Des DU de formation civile et civique soutenus par le ministère de l'Intérieur ont depuis été créés dans les universités de Bordeaux, de Paris I, de Paris XI, de Rennes, de Lille, de Nantes, de Toulouse, de La Réunion, de Lorraine, et au sein du Centre universitaire de Mayotte. L'ouverture d'un DU à distance, corollaire de l'obligation de diplôme pour les aumôniers rémunérés fixée par le décret de 2017, est prévue pour la rentrée 2017. Les universités publiques, conscientes des enjeux sociétaux de ces diplômes ne s'opposent plus à leur création. Au contraire, les initiatives émanant des universités publiques sont désormais 
plus nombreuses que les nouvelles créations envisagées dans le cadre de la politique de développement du ministère de l'Intérieur et du ministère de l'Enseignement supérieur et de la Recherche.

Le financement par le ministère de l'Intérieur des formations précitées, à raison de 15000 euros par diplôme et par année, a permis d'établir une carte nationale des $\mathrm{DU}$, ce qui était la condition préalable à un texte imposant aux aumôniers des services publics une formation civile et civique comme condition de leur rémunération. L'arrêté du 5 mai 2017 prévoit un mécanisme d'habilitation conjointe des formations par les ministres de l'Éducation nationale et de l'Intérieur, pour une durée de 5 ans, les demandes devant être transmises au Bureau central des cultes avant le 31 mai de chaque année. L'inscription sur la liste des formations devrait garantir un financement public, mais dans la mesure où le texte ne le dit pas expressément, la pérennité de ce soutien n'est absolument pas garantie. On notera que de son côté, la Fondation de l'islam de France a pris la décision de verser des bourses susceptibles de couvrir les frais d'inscription d'étudiants exerçant des fonctions de cadres religieux (aumôniers, imams).

\subsection{LE DIPLÔME ET LE CONTENU DES FORMATIONS}

Avec le décret du 3 mai 2017 et l'arrêté consécutif du 5 mai, les formations jusqu'ici mises en place à l'initiative des universités s'inscrivent dans un cadre réglementaire contraignant, qui fixe à la fois le contenu des formations et les droits liés à la délivrance du diplôme de formation civile et civique.

Les formations, indique l'article $1^{\text {er }}$ de l'arrêté, « comprennent au moins les trois enseignements suivants : $1^{\circ}$ Institutions de la République et laicité ; $2^{\circ}$ Grands principes du droit des cultes; $3^{\circ}$ Sciences humaines et sociales des religions ». Autrement dit, l'objectif poursuivi est triple :

- transmettre un socle de valeurs communes comprenant notamment la connaissance des institutions de la République, et en particulier du principe de laïcité et de ses applications (principe de neutralité et liberté de conscience et de culte ; égalité et non-discrimination ; présentation des politiques publiques en matière d'intégration);

- fournir des instruments en vue de faciliter la gestion des personnels et des institutions cultuelles et préparer les aumôniers à l'exercice de leurs fonctions dans le cadre d'une administration ou de l'armée, grâce à un enseignement 
de droit des cultes (régime des associations cultuelles, des édifices du culte, statut des ministres du culte et fiscalité des cultes), mais également de droit de la famille et de droit du travail en lien avec le fait religieux.

- faciliter la connaissance du fait religieux et des religions implantées sur le territoire français grâce à un enseignement de sciences humaines et sociales des religions (analyse sociologique des dynamiques des groupes religieux dans le contexte de sécularisation, histoire des évolutions religieuses en France).

Les enseignements sur les institutions de la République et les grands principes du droit des cultes représentent au moins 70 heures de cours sur un volume horaire total de 125 à 160 heures (art. $1^{\text {er }}$ de l'arrêté).

Ces diplômes, qui sont surtout organisés dans le cadre des facultés de droit, attirent également un public d'étudiants juristes souhaitant bénéficier d'un enseignement de droit des religions. L'inscription à la formation, qui ne peut être soumise à une condition de diplôme, est autorisée à la suite d'une évaluation du dossier d'inscription par le responsable du diplôme, éventuellement assisté par une commission et après convocation des candidats à un entretien. Actuellement, les responsables des diplômes de formation civile et civique se réunissent au moins une fois par an sur invitation du Bureau central des cultes du ministère de l'Intérieur en vue d'échanger leurs expériences et de structurer et maintenir un cadre commun aux diplômes : contenu des programmes, volume horaire, coût de la formation, modalités d'équivalence, organisation de l'enseignement à distance et harmonisation des règlements d'examen.

La nature de ces nouveaux diplômes de formation civile et civique est incertaine au regard de la distinction introduite par la loi Savary de 1984 entre les diplômes nationaux, enserrés dans une réglementation extrêmement rigoureuse, et les diplômes d'université qui relèvent de l'autonomie des universités. Ainsi que l'énonce l'article L. 613-2 du Code de l'éducation, outre les diplômes nationaux qu'ils sont autorisés à délivrer, " les établissements peuvent aussi organiser, sous leur responsabilité, des formations conduisant à des diplômes qui leur sont propres ou préparant à des examens ou des concours ». À l'évidence, les diplômes de formation civile et civique ne constituent pas des diplômes nationaux au sens de l'article L. 613-1. C'est en effet sous la forme de diplômes d'université qu'ils ont été créés initialement. Mais les dispositions de l'arrêté du 5 mai 2017 qui tout à la fois créent le diplôme, mettent en place une procédure interministérielle d'agrément et fixent des conditions pour leur délivrance, posent la question du respect de 
l'autonomie reconnue aux universités relativement à des diplômes qui leur sont propres. On relèvera ici que les formations pouvant être délivrées par les universités publiques, mais aussi par les établissements d'enseignement supérieur privés d'intérêt général (ce qui inclut l'enseignement supérieur confessionnel), ni l'arrêté ni le décret ne parlent de diplôme d'université, mais de diplôme (sans épithète). Quoi qu'il en soit, les dispositions essentielles relatives au diplôme figurent dans un simple arrêté interministériel, ce qui surprend quelque peu.

\section{LA FORMATION CIVILE ET CIVIQUE COMME CONDITION DE LA RÉMUNÉRATION}

Si la possession du diplôme de formation civile et civique ouvre droit pour les aumôniers à une rémunération de l'administration où ils interviennent, en revanche l'article 2 de la loi de 1905 ne crée aucune obligation de rémunération (2.1). Même s'il ne le dit pas expressément, le nouveau décret ne ferme pas la porte aux aumôniers non diplômés qui pourront continuer à exercer leur office religieux, mais à titre purement bénévole (2.2). Désormais, seuls les aumôniers diplômés pourront prétendre à une rémunération (2.3).

\subsection{L'ABSENCE D'OBLIGATION DE RÉMUNÉRER LES AUMÔNIERS}

Le régime juridique de l'aumônerie des services publics prend sa source dans l'article 2 de la loi de 1905, qui par exception à l'interdiction de financer les cultes autorise l'État et les collectivités publiques à inscrire à leur budget « les dépenses relatives à des services d'aumônerie et destinées à assurer le libre exercice des cultes dans les établissements publics tels que lycées, collèges, écoles, hospices, asiles et prisons ». L'énumération de l'article 2 n'étant pas exhaustive, les aumôneries ont été maintenues après la séparation dans ces lieux clos où se joue le salut des âmes que sont les institutions scolaires, la prison, l'hôpital, mais également l'armée que le législateur de 1905 avait omis de citer. Seulement avec la séparation, l'existence de l'aumônerie ne procède plus de cette logique coopérative qui prévalait au temps de la reconnaissance, elle n'est plus désormais que le moyen d'« assurer le libre exercice des cultes » des agents ou des usagers privés d'un accès usuel aux prestations religieuses. De cela, il est résulté une désinstitutionnalisation de 
l'aumônerie, plus ou moins importante selon les services concernés, mais sans que jamais le principe de son existence ne soit remis en cause. Au contraire, non seulement les services d'aumônerie ne seront pas supprimés, mais leur financement sera maintenu, à l'exception de l'aumônerie scolaire, où de son caractère facultatif on conclura à la suppression définitive de tout financement public.

Depuis la séparation, les aumôneries des quatre services publics se sont réorganisées sur des bases juridiques extrêmement dissemblables, et chacune d'elles obéit à un régime propre caractérisé toutefois par un certain nombre de similitudes, concernant en particulier la procédure de nomination qui suppose une investiture préalable de l'autorité religieuse de rattachement, ou encore le droit pour celle-ci de retirer son agrément à tout moment. Mais jamais, et le fait mérite d'être souligné, le pouvoir réglementaire n'était jusqu'ici intervenu pour imposer des dispositions communes à l'ensemble des aumôneries existantes. C'est à ce particularisme que vient mettre fin le nouveau décret du 3 mai 2017 relatif aux aumôniers militaires, hospitaliers et pénitentiaires et à leur formation civile et civique. Ce décret prescrit que les aumôniers militaires, hospitaliers et pénitentiaires rémunérés recrutés à partir du $1^{\text {er }}$ octobre 2017 devront obligatoirement être titulaires d'un diplôme de formation civile et civique ou, à défaut, s'engageront à l'obtenir dans un délai de deux ans après leur recrutement. L'arrêté du 5 mai 2017 qui le complète fixe les modalités d'agrément des formations qui seront habilitées à délivrer ces diplômes. L'exclusion de son champ d'application de l'aumônerie scolaire ne change rien au caractère général de ce décret, dont l'objet est d'imposer une formation civique aux seuls aumôniers rémunérés sur fonds publics, ce qui exclut de facto les aumôneries scolaires où l'on n'a pas voulu réintroduire une rémunération publique qui a disparu aux lendemains de la séparation.

\subsection{LE MAINTIEN DE LA CATÉGORIE DES AUMÔNIERS NON RÉMUNÉRÉS}

Outre qu'il se distingue par son caractère de généralité, ce décret retient l'attention en ce qu'il impose une obligation de formation érigée en condition de l'accès aux fonctions d'aumônier rémunéré. Jusqu'ici, les aumôniers qu'ils soient bénévoles ou rémunérés étaient recrutés par l'autorité administrative sur proposition des autorités religieuses, et cette proposition constituait d'ailleurs le critère quasi exclusif de la nomination. L'administration était en outre liée par la décision de retrait des autorités 
religieuses ${ }^{1}$. À propos des aumôniers des services publics dont il avait étudié le statut, Georges Dole a pu parler de "double investiture ", à la fois civile (nomination) et religieuse (désignation). Ce qui change avec ce nouveau décret, c'est que la nomination par l'autorité administrative est assortie désormais d'une condition de capacité civile et civique. Cette exigence de formation vise à garantir que les aumôniers sont imprégnés des valeurs de la République et en particulier du principe de laïcité, et de surcroît qu'ils ont la connaissance du cadre administratif dans lequel ils sont appelés à intervenir. Mais si l'on pousse à son terme la logique du dispositif, alors il devient manifeste que l'ambition du projet est bel et bien la transmission aux malades, aux prisonniers et aux soldats qui n'en seraient pas naturellement imprégnés des valeurs communes acquises au cours de la formation, en sorte que c'est la fonction même des aumôniers qui se trouve en quelque sorte dédoublée par la création d'une aumônerie « laiqque» investie d'une fonction éducative. Une telle logique est confirmée par le fait que le dispositif a été conçu en vue notamment de permettre le recrutement d'aumôniers musulmans chargés de lutter contre les phénomènes de radicalisation dans les prisons.

La principale difficulté que pouvait présenter, du point de vue de l'économie générale de la réforme, une telle exigence de diplôme, était qu'en restreignant une liberté de désignation jusqu'ici inconditionnée, l'ingérence de l'autorité publique ne porte atteinte à la liberté de religion. C'est la raison pour laquelle, malgré une certaine imprécision du décret sur ce point, le diplôme n'est en aucun cas la condition d'accès aux fonctions d'aumônier, mais la condition de la seule rémunération publique. Autrement dit, il existera donc à l'avenir au sein des services publics concernés deux catégories d'aumôniers, d'une part les aumôniers diplômés/rémunérés par les pouvoirs publics, et d'autre part les aumôniers qui n'étant pas diplômés interviendront à titre gratuit ou seront rémunérés par leur culte. Pour ces derniers, ce sont les dispositions jusqu'ici appliquées qui continueront de l'être, à moins que les ministères concernés n'adaptent leurs pratiques à la suite du décret. On notera toutefois sur cette question de la distinction du caractère gratuit ou rémunéré des fonctions que si la possession d'un diplôme de formation civile et civique est à l'avenir la condition nécessaire de la rémunération, en revanche elle n'en est en aucun cas la condition suffisante. S'il ne sera plus possible à l'avenir de rémunérer des aumôniers

1. CE, 17 oct. 1980, Pont, n 13567, Rec. CE, p. 374; AJDA mai 1981, p. 256, concl. Labetoulle. 
non diplômés, en revanche rien dans le décret ne vient obliger les administrations concernées à rémunérer les aumôniers diplômés, pas plus qu'elle ne les oblige à les recruter.

\subsection{L'IMPOSITION DU DIPLÔME COMME CONDITION DE LA RÉMUNÉRATION}

Si une telle obligation de diplôme a été imposée à l'ensemble des aumôniers rémunérés, c'est pour d'évidentes raisons de non-discrimination à l'égard des aumôniers musulmans. Cette situation a d'ailleurs suscité des protestations de la part de l'Église catholique.

La justification d'une telle exigence de formation, pour le gouvernement à l'origine du dispositif, réside dans le fait que si les aumôniers rémunérés sont au service de leurs coreligionnaires, ils participent également au fonctionnement quotidien de ces institutions fortement structurées (armée, hôpital, prison) dans lesquelles ils doivent être parfaitement intégrés. Ainsi les aumôniers hospitaliers ne font-ils pas partie des équipes soignantes, mais ils collaborent néanmoins avec elles dans une perspective de prise en charge globale de la personne accompagnée. Une formation aux institutions de la République française, au droit des religions et au pluralisme religieux est donc justifiée sous cet angle. La formation exigée n'est pas de nature religieuse ou théologique, elle vise à faciliter l'intégration dans une administration ou dans les services de l'armée. D’une manière générale, le recrutement de personnels rémunérés par l'État ou des personnes publiques est sauf exception soumis à une condition de diplôme, et en ce sens le dispositif n'a rien d'exorbitant. Il reste que pour des cultes dont les aumôniers bénéficiaient jusqu'ici d'une rémunération sans condition particulière, et ne posaient aucun problème d'intégration, l'exigence d'un diplôme pour les candidats futurs est une source de préoccupation légitime. Enfin, il convient de ne pas oublier que le régime de l'aumônerie est ouvert à tous les cultes, ainsi qu'a pu le rappeler le Conseil d'État dans un important arrêt du 16 octobre 2013, Garde des Sceaux, ministre de la Justice et des Libertés $c / m$. $n$... et autres. Cet arrêt qui statue sur plusieurs recours exercés par l'Association des Témoins de Jéhovah est d'un double intérêt pour ce qui concerne la question de la nomination des aumôniers. D'une part, les cultes dits « minoritaires » ne peuvent être exclus a priori du dispositif de formation ni de l'accès aux fonctions d'aumôniers rémunérés, au motif notamment de l'insuffisance du nombre des détenus se revendiquant de la confession en cause. D'autre part, l'existence d'un régime d'agrément pour les aumôniers (en l'espèce dans les prisons) 
ne saurait impliquer, en l'état de la législation et de la réglementation, « que les aumôniers agréés auprès des établissements pénitentiaires ne pourraient pas être bénévoles ». Pour dire les choses autrement, l'institutionnalisation dont l'aumônerie est l'objet ne doit en aucun cas servir de fondement à un refus d'accéder aux prestations religieuses. C'est pour cela également que l'aumônerie à titre bénévole ne peut être supprimée purement et simplement.

\section{UNE HARMONISATION TRÈS PARTIELLE DU RÉGIME DE L'AUMÔNERIE}

Le décret du 3 mai 2017 ne remet pas en cause les spécificités propres à chaque catégorie d'aumônerie (3.1). Il prévoit par ailleurs un certain nombre de dispositions particulières qu'il conviendra de présenter pour terminer (3.2).

\subsection{LES DISPOSITIONS SPÉCIFIQUES AUX DIFFÉRENTES CATÉGORIES D'AUMÔNERIES}

Contrairement à ce que suggère la mise en place d'une formation commune aux aumôniers des hôpitaux, des prisons et des armées, l'unification du régime de l'aumônerie reste très incomplète. D'une part en effet, l'aumônerie des collèges et des lycées n'est pas visée par le nouveau décret. D'autre part, les spécificités des trois autres aumôneries ne sont pas remises en cause.

\section{L'AUMÔNERIE SCOLAIRE}

Si, pour la première fois depuis 1905, un texte unique réunit sous un même chapeau plusieurs catégories d'aumônerie, il reste que l'aumônerie des collèges et des lycées n'est pas visée par le nouveau dispositif, parce qu'elle ne fait l'objet d'aucune prise en charge publique ${ }^{2}$. Les textes adoptés sur le fondement de l'article $1^{\text {er }}$ de la loi Debré ${ }^{3}$ visaient à faciliter l'ouverture d'aumôneries lorsqu'une demande émanée des parents en était faite ${ }^{4}$. Mais ce

2. Décret du 22 avril 1960, art. 8 (codifié C. éduc., art. R. 141-7) : « Les frais d'aumônerie sont à la charge des familles, sous réserve de l'application des dispositions de l'article 2 de la loi du 9 décembre 1905 concernant la séparation des Églises et de l'État. »

3. «L'État prend toutes dispositions utiles pour assurer aux élèves de l'enseignement public la liberté des cultes et de l'instruction religieuse» (codifié C. éduc., art. L. 141-2).

4. La circulaire $\mathrm{n}^{\circ}$ 88-112 du 22 avril 1988 prescrivait, à propos de l'appréciation, par le recteur, de l'opportunité d'ouvrir une aumônerie, que « la règle générale doit être d'accorder satisfaction aux vœux des demandeurs, même si ceux-ci ne représentent qu'un très faible pourcentage de l'effectif total de l'établissement. Un refus, en effet, leur porte préjudice, 
libéralisme s'est trouvé pris en défaut par l'adoption de la loi du 15 mars 2004 interdisant aux élèves le port de signes ou de tenues manifestant ostensiblement une appartenance religieuse, en sorte que depuis lors les demandes de création d'aumônerie nouvelle sont refusées de manière quasi systématique afin d'éviter la création d'aumôneries musulmanes. Autrement dit, dans les collèges et les lycées l'aumônerie n'a pas bénéficié de ce regain de faveur qu'elle connaît aujourd'hui auprès des pouvoirs publics.

\section{L'AUMÔNERIE MILITAIRE}

Pour des raisons évidentes, liées notamment au fait qu'elle s'adresse à des agents soumis à un statut et non comme les autres aumôneries aux « usagers », l'aumônerie militaire est l'objet d'une forte institutionnalisation ${ }^{5}$. Ainsi que l'énonce le décret $\mathrm{n}^{\circ}$ 2008-1524 du 30 décembre 2008 modifié relatif aux aumôniers militaires, les aumôniers militaires sont des militaires servant en vertu d'un contrat à durée déterminée et renouvelable. Tenant compte de l'existence de ces contrats, le nouveau décret du 3 mai 2017 se contente d'ajouter aux trois conditions imposées par le décret de 2008 pour souscrire un contrat d'aumônier militaire ${ }^{6}$ une condition nouvelle consistant, pour les aumôniers d'active, à être titulaire d'un diplôme de formation civile et civique.

\section{L'AUMÔNERIE DES ÉTABLISSEMENTS HOSPITALIERS}

Dans les établissements hospitaliers, c'est une simple circulaire ministérielle de $2006^{7}$ qui organise le régime de l'aumônerie, son aménagement concret relevant de la compétence des responsables d'établissement hospitalier. La circulaire prescrit que la fonction d'aumônier peut être assurée, de façon permanente ou temporaire, soit par des aumôniers rémunérés ${ }^{8}$ recrutés en qualité de contractuels, soit par des aumôniers bénévoles. Les aumôniers rémunérés sont des agents publics non titulaires affectés dans

alors que la création du service de l'aumônerie ne nuit en rien aux convictions ni à la liberté de conscience des autres membres de la communauté scolaire ».

5. Les textes organisant l'aumônerie militaire ont été complètement refondus compte tenu de la création, en 2005, d'une aumônerie militaire musulmane.

6. $1^{\circ}$ Être en règle au regard des obligations du Code du service national ; $2^{\circ}$ N'avoir aucune mention au bulletin $n^{\circ} 2$ du casier judiciaire qui soit incompatible avec l'exercice des fonctions auxquelles il est postulé ; $3^{\circ}$ Être titulaire du baccalauréat de l'enseignement secondaire ou d'un titre reconnu équivalent.

7. Circulaire DHOS/P1/2006/538 du 20 décembre 2006.

8. La rémunération est fixée selon la grille indiciaire de l'échelle 5 de rémunération des agents de catégorie $\mathrm{C}$. 
un emploi d'agent contractuel, et soumis en tant que tels aux dispositions générales applicables aux agents contractuels des établissements hospitaliers ${ }^{9}$. Le décret du 3 mai 2017 qui impose aux aumôniers contractuels la possession du diplôme de formation civile et civique ne modifie pas ce dispositif, auquel il donne un fondement réglementaire. La seule modification, et elle est significative, réside dans le fait que les contrats dont la durée était de 3 ans minimum renouvelables, sont désormais «à durée déterminée ou indéterminée ». Les contrats à durée déterminée, d'une durée maximale de trois ans, ne peuvent être renouvelés que dans la limite d'une durée maximale de 6 ans, le renouvellement à l'issue de cette durée ne pouvant intervenir « que par décision expresse et pour une durée indéterminée ». L'entrée en vigueur du nouveau décret rend nécessaire la réécriture de la circulaire de 2006 dont un certain nombre de dispositions ont été rehaussées dans le décret. Par ailleurs, la circulaire contient une disposition prescrivant que les aumôniers bénévoles « devront cependant, pour être autorisés à exercer leur mission, remplir les mêmes conditions que ceux qui sont rémunérés ». Avec l'entrée en vigueur de l'exigence de diplôme, cette disposition devient caduque.

\section{L'AUMÔNERIE DES PRISONS}

C'est un décret ( $n^{\circ}$ 2010-1634) du 23 décembre 2010, pris en application de la loi pénitentiaire du 24 novembre 2009, qui fixe les dispositions relatives à l'assistance aux détenus. Ces dispositions ont été fondues dans la partie réglementaire du Code de procédure pénale ${ }^{10}$. Un autre décret $\left(n^{\circ}\right.$ 2010-1635) du même jour organise un régime d'agrément des aumôniers par le directeur interrégional des services pénitentiaires après avis du préfet du département dans lequel se situe l'établissement visité, sur proposition de l'aumônier national du culte concernéll. Le décret du 3 mai 2017 qui complète l'article D. 439 ne remet pas en cause ce mécanisme d'agrément, mais il énonce $q^{\prime}$ '« en vue de leur permettre d'assurer les missions qui leur sont confiées, une indemnité forfaitaire peut être allouée aux aumôniers agréés », et fait de la possession du diplôme de formation civile et civique une condition de la rémunération. Il existera donc à l'avenir deux catégories d'aumôniers agréées,

9. La circulaire renvoie à l'article 2 de la loi no $86-33$ du 9 janvier 1986 portant dispositions statutaires relatives à la fonction publique hospitalière et au décret du 6 février 1991 relatif aux agents contractuels des établissements hospitaliers.

10. CPP, art. R. 57-9-3 à 7.

11. CPP, art. D. 439. 
ceux qui titulaires d'un diplôme pourront bénéficier d'une rémunération, et ceux qui ne l'étant pas exerceront leurs fonctions à titre gratuit.

À la différence des aumôniers militaires et hospitaliers, les aumôniers pénitentiaires ne bénéficient donc pas d'un contrat et ils n'ont pas la qualité d'agents publics, ce que n'ont pas manqué de déplorer les aumôniers pénitentiaires pour qui le nouveau décret ne représente guère qu'une contrainte supplémentaire, sans apporter d'amélioration à leur condition, et ce alors que le nouveau dispositif avait été pensé pour eux, en particulier les aumôniers musulmans confrontés aux phénomènes de radicalisation dans les prisons.

\subsection{L'APPLICATION DU DÉCRET DANS LE TEMPS. LES DISPOSITIONS TRANSITOIRES}

La date d'entrée en vigueur du décret du 3 mai 2017 a été fixée au $1^{\mathrm{er}}$ octobre 2017. Afin d'empêcher le tarissement des recrutements à compter de cette date, le décret prévoit la possibilité de recruter des aumôniers non titulaires du diplôme à la condition qu'ils s'engagent à obtenir le diplôme dans les deux années suivant leur recrutement ou leur agrément. Le contrat n'est pas renouvelé en cas de non-obtention du diplôme.

En ce qui concerne les aumôniers titulaires d'un contrat antérieur à la date du $1^{\text {er }}$ octobre 2017, il a été décidé de ne pas exiger la possession du diplôme à l'occasion des renouvellements ultérieurs. En ce qui concerne l'agrément des aumôniers de l'administration pénitentiaire, il est accordé sans limitation de durée, la limite d'âge entraînant son retrait étant fixée à 75 ans (Code de procédure pénale, art. D. 439). Les situations acquises ne sont donc pas remises en cause par le décret.

Quant à l'application du décret aux collectivités d'outre-mer, elle est subordonnée à la condition qu'un diplôme puisse y être obtenu localement, y compris à distance. 